\title{
CONTAMINACIÓN POR ELEMENTOS MENORES Y POSIBLES SOLUCIONES
}

\section{MINOR ELEMENT CONTAMINATION AND POSSIBLE SOLUTIONS}

Rodrigo Lora Silva ${ }^{1}$

\section{RESUMEN}

En este artículo, se analizan algunos problemas causados por el exceso de los micronutrientes esenciales Fe, $\mathrm{Cu}, \mathrm{Mn}, \mathrm{Zn}, \mathrm{Ni}, \mathrm{B}, \mathrm{Mo}$ y Cl, y otros elementos no esenciales para hombres y animales, como cadmio, plomo, cromo, arsénico y selenio. Se consideran los factores que afectan la solubilidad de estos elementos, como reacciones redox, reacción del suelo, complejos orgánicos, reacciones de precipitación, adsorción superficial y reacciones biológicas. Se examinan, igualmente, los síntomas visuales, el análisis foliar y el análisis de suelos, como herramientas de diagnóstico de la toxicidad de elementos menores. Finalmente, se hace referencia a las posibles soluciones a la contaminación y la toxicidad por microelementos, tanto de manejo preventivo como de recuperación.

Palabras clave: micronutrientes, contaminación, síntomas visuales, diagnóstico, recuperación.

\section{SUMMARY}

In this article the problems due to the excess of the essential micronutrients $\mathrm{Fe}, \mathrm{Cu}, \mathrm{Mn}, \mathrm{Zn}, \mathrm{Ni}, \mathrm{B}, \mathrm{Mo}$ and $\mathrm{Cl}$ are analyzed, as well as those caused by other non

1 Ingeniero Químico, M.Sc. Docente Investigador, Carrera de Ingeniería Agronómica, U.D.C.A. Dirección para Correspondencia: Calle 222 No. 55-37, Bogotá, D.C. essential elements such as cadmium, lead, chromium, arsenic and selenium. The factors that affect the solubility of these elements as redox reactions, soil reaction, organic complexes, precipitation reactions, superficial adsorption and biological reactions are considered. The visual symptoms, foliar and soil analysis are examined as tools to diagnose the micronutrients toxicity. Finally, reference is made of the possible solutions for contamination and toxicity of microelements for both preventive and recuperation management.

Key words: micronutrients, visual symptoms, diagnosis, contamination, recuperation.

\section{INTRODUCCIÓN}

Dentro de los elementos esenciales para la planta existen ocho que son requeridos en cantidades pequeñas, por lo cual, se les denomina elementos menores o micronutrimentos. Estos elementos son el boro (B), cobre $(\mathrm{Cu})$, hierro $(\mathrm{Fe})$, manganeso $(\mathrm{Mn})$, zinc $(\mathrm{Zn})$, niquel (Ni), molibdeno (Mo) y cloro (Cl). Existen algunos elementos cuya esencialidad no ha sido probada, pero cuya presencia en algunos casos produce efectos benéficos, tales como el silicio ( $\mathrm{Si}$ ), yodo (I) y sodio (Na); o en ciertos casos el elemento es necesario para que se efectúe un determinado proceso, tal como ocurre con el cobalto (Co), en la fijación simbiótica del nitrógeno. Igualmente, la planta puede tomar algunos elementos que no desempeñan ninguna función de beneficio, pero que pueden llegar a ser tóxicos para plantas y animales cuando se 
sobrepasan ciertos límites. Tal es el caso del cadmio (Cd), mercurio $(\mathrm{Hg})$, plomo $(\mathrm{Pb})$, selenio $(\mathrm{Se})$, arsénico (As) y cromo (Cr), entre otros (Lora, 1998; 2001; Brown et al. 1987; Marschner, 2003; Méndez et al. 2000; Luján, 2005; Reiman et al. citado por Matamoros, 2003). El riego con aguas contaminadas ha producido toxicidad de $\mathrm{B}, \mathrm{Cd}, \mathrm{Pb}$ y $\mathrm{Cr}$, principalmente y ha afectado la salud de hombres y animales, en cuando se consumen cultivos sensibles o acumuladores, como cebolla, ajo, lechuga, remolacha, papas y nabos (Lucho et al. 2005).

\section{Formas químicas de los micronutrimentos que pueden ser absorbidas por las plantas:}

Para conocer el problema de contaminación por exceso de micronutrimentos en el medio de crecimiento de la planta, se requiere tener información relacionada con las formas químicas que pueden ser tomadas por ellas. De acuerdo con Munevar (1999), los cationes Fe, Cu, Mn, $\mathrm{Z}$ y Ni son tomados o absorbidos en forma iónica. Por su parte, los elementos $\mathrm{B}, \mathrm{Mo} \mathrm{y} \mathrm{Cl}$, la planta los absorbe en forma aniónica o en forma molecular, por tanto es importante tener en cuenta la química de los cationes y los aniones respecto a las propiedades químicas del suelo (Lora, 2003), lo cual puede permitir un manejo adecuado de la toxicidad.

La solubilidad de los micronutrimentos está asociada con numerosas reacciones, entre las cuales se destacan:

Reacciones Redox: La disponibilidad de elementos como el Fe y el Mn está controlada en gran parte por reacciones de óxido reducción. Las formas oxidadas de estos elementos son poco solubles y por tanto no disponibles, en contraste con las formas reducidas. Factores como humedad y aireación controlan estas reacciones en el suelo e influyen en su disponibilidad y, en algunos casos, en el exceso o contaminación por parte de estos dos nutrimentos. Recalca Lora (1999), que en el cultivo de arroz de riego o inundación juega papel importante este factor, especialmente para el Fe y el Mn, tal como ocurre en algunos suelos de los Llanos Orientales de Colombia.

Complejos Orgánicos: La materia orgánica (MO) forma compuestos estables con los iones metálicos, esto es, con el cobre, el manganeso, el hierro, el zinc y el níquel. La estabilidad de los complejos sigue el siguiente orden:

$$
\mathrm{Cu}^{++}>\mathrm{Zn}^{++}=\mathrm{Fe}^{++}>\mathrm{Mn}^{++}>\mathrm{Ni}^{++}
$$

Los principales efectos de los agentes complejantes derivados de la materia orgánica corresponden a mantener en solución, como complejos solubles, elementos menores que de otra forma se precipitan, transformar micronutrimentos de la fase sólida en formas solubles, servir como vehículo para el movimiento de estos elementos hacia la raíz y convertir, en formar no asimilables, micronutrimentos que se hallan en el suelo. Además, los complejos orgánicos solubles pueden colaborar para que algunos micronutrientes contaminantes se pierdan por lixiviación o para inducir deficiencias de algunos micronutrimentos esenciales para la planta (Matamoros, 2003; Wong et al. 2006).

La capacidad de los ácidos húmicos y fúlvicos para formar complejos estables con iones metálicos, se puede atribuir a su alto contenido de grupos funcionales que contienen oxígeno, tales como $\mathrm{COOH}^{-}, \mathrm{OH}^{-}$, fenólicos, alcohólicos y $\mathrm{C}=\mathrm{O}$. Los ácidos fúlvicos tienen pesos moleculares más bajos que los húmicos, por lo cual, se cree que cumplen función muy importante en el mantenimiento de los micronutrientes catiónicos en la solución del suelo. Los ácidos húmicos forman compuestos más insolubles (Burbano, 1998; Lora, 1999; Zapata, 2005).

Reacciones de Precipitación: La formación de precipitados insolubles es uno de los fenómenos que más afectan la disponibilidad de los micronutrientes. Estos precipitados incluyen óxidos, hidróxidos, fosfatos, carbonatos y molibdatos. En general, la solubilidad disminuye al aumentar el $\mathrm{pH}$ con excepción de los molibdatos. Algunos autores, como Lora (1999), Tisdale et al. (1993) y Barrera (2003), mencionan que puede también haber insolubilización por coprecipitación con compuestos como $\mathrm{Al}(\mathrm{OH})_{3}, \mathrm{CaCO}_{3}$ y $\mathrm{Fe}(\mathrm{OH})_{3}$, quedando así el elemento ocluido fuera del alcance de la planta.

Adsorción Superficial: La adsorción superficial o retención en la superficie de coloides u otros materiales, depende del pH. En los cationes aumenta con el pH, mientras que la de los aniones disminuye, esto es, en condiciones de acidez, la adsorción aniónica puede ser elevada. La mayor adsorción del Cu y el Zn por la montmorillonita ocurre a $\mathrm{pH}$ neutro o alcalino bajo las formas de $\mathrm{CuOH}^{+}$y $\mathrm{ZnOH}^{+}$, presentes a $\mathrm{pH}$ altos. A medida que aumentan las condiciones reductoras del medio, se presenta un incremento de los iones $\mathrm{Fe}^{++}$y $\mathrm{Mn}^{++}$ y, por tanto, mayor absorción por parte de las plantas. Se debe tener en cuenta que si el $\mathrm{pH}$ es mayor que 7 
podría haber precipitación de hidróxidos de Fe y Mn, reduciéndose la disponibilidad de los dos nutrimentos, como podría ocurrir en ciertas etapas de suelo inundado en arroz bajo riego.

Para B, la forma iónica adsorbida por los coloides del suelo es $\mathrm{B}(\mathrm{OH})_{4}$, adsorbida principalmente por el $\mathrm{Al}(\mathrm{OH})_{3}$. En general, las arcillas de tipo micáceo tienen gran habilidad para adsorber $B$, según la siguiente secuencia:

\section{Vermiculita $>$ Caolinita $>$ Montmorillonita}

$\mathrm{El} \mathrm{pH}$, al cual ocurre la máxima adsorción de $\mathrm{B}$, es dependiente del coloide, así: 5,5 a 7,0 para $\mathrm{Al}_{2} \mathrm{O}_{3}, 7,0$ para $\mathrm{Al}(\mathrm{OH})_{3}$ y 8 a 9 para $\mathrm{Fe}_{2} \mathrm{O}_{3}$ y arcillas (Boyd \& Knezek, citados por Lora, 1999).

La adsorción del anión molibdato, se puede deber a enlaces covalentes entre los iones molibdato y los hidróxidos superficiales de coloides, o por intercambio aniónico entre $\mathrm{OH}^{-}$y $\mathrm{MoO}_{4}$. Posteriormente, ocurre precipitación de molibdato de hierro o aluminio (Motta, 1976; Bohn et al. 2001; Fassbender, 1982), de acuerdo a la siguiente reacción:

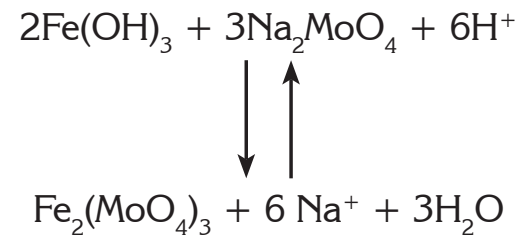

De acuerdo con esta reacción es necesario un medio ácido para que ocurra la fijación de molibdeno. La capacidad de los coloides para adsorber el elemento sigue el siguiente orden:

Óxidos de Hierro $>$ Óxidos de Aluminio $>$ Halloisita $>$ Nontronita $>$ Caolinita

Reacciones Biológicas: Los procesos microbiológicos están involucrados en las reacciones que intervienen en la disponibilidad de los micronutrimentos. Los microorganismos pueden afectar la disponibilidad de los elementos menores liberando iones inorgánicos durante la descomposición de la $\mathrm{MO}$, esto es, durante la mineralización de los materiales orgánicos e inmovilizando los micronutrimentos por incorporación en las estructuras microbiales. Además por oxidación, por reducción y por ocasionar cambios del $\mathrm{pH}$ y/o potencial de óxido-reducción, afectando la forma y la solubilidad de los microelementos. Cualquier factor que afecte la actividad de los microorganismos estará igualmente articulado a la disponibilidad de los micronutrimentos del suelo. En general, se reduce la actividad de las bacterias nitrificantes y la actividad de los microorganismos descomponedores de la MO. Entre estos factores, se destacan la reacción del suelo, los micronutrimentos y los metales pesados, los macronutrimentos, el contenido de agua del suelo, las condiciones de oxidación y de reducción, la temperatura y la materia orgánica, entre otros (Burbano, 1998; Lora, 2003, Kirilkaya et al. 2004; Frey et al. 2006; Dai et al. 2004).

En la Figura 1, aparecen las reacciones que afectan la concentración de micronutrientes en la solución del suelo. La mayoría de los nutrimentos esenciales o no esenciales son afectados por estas reacciones con relación a concentración en la solución y, por tanto, a su disponibilidad para la planta que, en muchos casos, puede llegar a niveles tóxicos (Lindsay, 1973).

García (1998) menciona como factores para una aplicación racional de fertilizantes de elementos menores, la velocidad y la capacidad de reemplazo en la solución del suelo, la concentración en la solución del suelo, el movimiento de los nutrimentos a la superficie de la raíz, el cual se realiza por el flujo de masa, la difusión y la interceptación radicular. Además, varios autores, como Brown et al. (1972), Mengel \& Kirkby (1979), Lora (1994) y Tamayo \& Hincapié (2002), indican que se deben tener en cuenta algunos factores que afectan la habilidad de la planta para tomar o absorber micronutrimentos y entre estos, se pueden citar la atmósfera y la temperatura del suelo, el antagonismo iónico, las sustancias tóxicas, la actividad microbial, los impedimentos mecánicos y la zona radicular.

La toxicidad es el resultado de la absorción de algún elemento y de su acumulación en una cantidad tal que causa daño en la planta. La magnitud del daño depende, según IFDC (1985), Marschner (2003) y Lora (2003), del tiempo, de la concentración, de la clase y del uso del agua y de la susceptibilidad del cultivo. Algunos pastos, híbridos de maíz y leguminosas, como la alfalfa, pueden tolerar situaciones elevadas de aluminio o altas cantidades de boro y cloro (Maas, citado por García, 1994). 


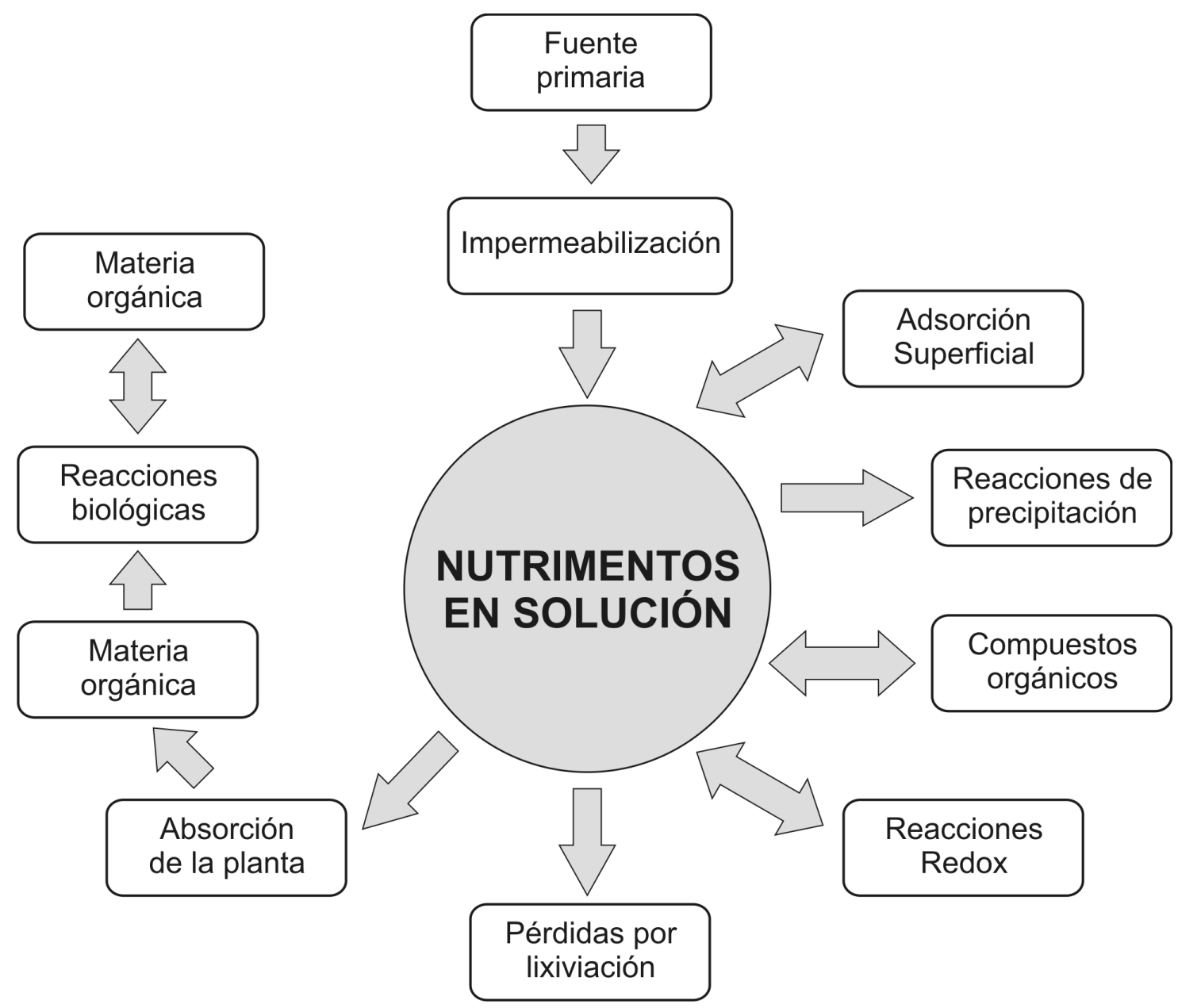

Figura 1. Reacciones asociadas con la solubilidad de los micronutrientes (adaptado de Linsay, 1973)

\section{Diagnóstico de la toxicidad por micronutrimentos}

Para diagnosticar problemas de toxicidad por micronutrimentos, se requiere que la persona encargada de hacer el diagnóstico conozca el cultivo. Se deben considerar algunas características, tales como la rizofera, la temperatura, el $\mathrm{pH}$, las plagas, las enfermedades, la humedad del suelo, los problemas de salinidad y sodicidad, los daños por herbicidas, la identificación y control de malezas, labranza, híbrido o variedad, la densidad de siembra, el manejo del agua, la época de siembra, la edad del cultivo y la fertilización donde se considere fuentes, dosis, método y época de aplicación de los fertilizantes (Molina E Frye, 1982; Tisdale et al. 1993; Lora, 1994; 2003; 2005; Mejía, 1998; Lora, 2005).
El conocimiento adecuado de las condiciones o factores antes mencionados, ayuda a determinar si la presencia de un problema está provocando deficiencia o toxicidad de uno o varios elementos. De esta manera, se puede determinar la deficiencia o la toxicidad de elementos menores o de elementos traza contaminantes en las plantas, utilizando herramientas de diagnóstico, tales como síntomas visuales, análisis foliar y análisis de suelos. Lo más recomendable es utilizar las tres, ya que ellas se complementan para realizar un diagnóstico más confiable (Marín, 1986; INPOFOS, 1993).

\section{SÍNTOMAS VISUALES}

La apariencia de una planta ha sido usada como una ayuda para determinar el estado de "salud" de ésta. Se 
debe tener en cuenta que el síntoma no solo puede indicar deficiencia o toxicidad, sino también la presencia de una enfermedad, al ataque de plagas, problemas de heladas, toxicidad por herbicidas, viento, granizo o implementos de campo y a deficiencia o exceso de agua. Por tanto, no siempre es fácil determinar exactamente cuál es el problema que indica la planta con el síntoma. Para hacer un adecuado diagnóstico, se requiere estudio, experiencia y, sobre todo, conocer muy bien cuál es el aspecto de una planta saludable. Es necesario tener un amplio conocimiento de los problemas que afectan los cultivos y hacer una evaluación adecuada de los mismos. Para diagnosticar por síntomas deficiencias nutricionales, se debe conocer la localización de los síntomas de los elementos móviles e inmóviles. Los síntomas de toxicidad pueden aparecer en toda la planta o en las hojas viejas, primordialmente. Conforme a lo mencionado por Lora (1994), Medina (2003), Marschner (2003) y García (1998) es importante considerar los factores relacionados con la toxicidad de los micronutrimentos, de la siguiente manera:

Boro: La toxicidad por boro puede llegar a ser un problema más grave que la deficiencia. Como lo mencionan Keren \& Bingham (1985), Turner (1978), Lora (1989), Garavito \& León (1978), García et al. (1999) y Lora et al. (2002a) podría existir toxicidad en suelos de regiones áridas o semiáridas que reciben aguas de drenaje provenientes de sedimentos marinos ricos en boro, en suelos regados con agua con más de $0,5 \mathrm{ppm}$ de boro para cultivos susceptibles y más de $4 \mathrm{ppm}$ para cultivos tolerantes, en suelos altamente fertilizados con boro y en suelos salinos o sódicos. El aguacate, el ciruelo y la vid son sensibles. Alfalfa, repollo, papa y tabaco son semitolerantes; algodón, remolacha y ajonjolí son tolerantes.

Cobre: Jiménez (1983), Ortiz (1998), Matamoros (2003) y Mejía (1998) mencionan la ocurrencia de toxicidad en suelos arenosos podzólicos bajos en $\mathrm{MO}$, la acumulación de cobre por uso continuo de fungicidas a base del elemento o por fertilización continua con el elemento; igualmente en suelos ricos en cobre por actividades urbanas e industriales (Abbas et al. 2006).

Hierro: El Fe puede causar problemas, como lo afirman Marschner (2003), Escobar (1985), Castillo (1985), Acosta (2003) y Bernal E Gómez (2004), en oxisoles o ultisoles ricos en hierro y bajos en manganeso, bajo condiciones reducidas, como en arroz de riego, en suelos ácidos a fuertemente ácidos o debido a la aplicación continua y dosis elevadas de fertilizantes de hierro.

Manganeso: Grandett (1979), García (1981), Villar E Lora (1992), Bernal $\&$ Triana (1980), Acosta (2003), Guerrero E Burbano (1979) y García (1998) consideran las condiciones reducidas, como en arroz de riego, la fertilización continua con manganeso, los suelos ácidos y además con bajo contenido de $\mathrm{Fe}$, los altos contenidos de manganeso total y cambiable y la aplicación continua de fungicidas con manganeso, como características que favorecen la toxicidad por Mn. Contenidos iguales o mayores de 150ppm en el suelo redujeron el rendimiento y la calidad de la fresa (Casierra E Poveda, 2005).

Molibdeno: De acuerdo a Motta (1976), Jiménez (1983), Lora et al. (2004), Agreda et al. (1992), Lora (1978) e Higuita E Lora (1979), la toxicidad de Mo se manifiesta en suelos alcalinos orgánicos, en suelos derivados de deposiciones volcánicas recientes, en suelos esqueléticos de colina derivados de arcillolitas y areniscas, bajo la presencia de pizarras. Además, a causa de la aplicación continua de fertilizantes de molibdeno y en suelos ya sea con elevados contenidos de fósforo u orgánicos pobremente drenados, se puede presentar toxicidad.

Zinc: Gutiérrez (1974), Lora et al. (2002b) y Marschner (2003) mencionan la posible toxicidad en suelos ácidos o ligeramente ácidos, en turbas ácidas, en suelos contaminados con zinc de operaciones mineras o derivados de rocas y materiales altos en $\mathrm{Zn}$. Consideran también la aplicación continua y elevada de fertilizantes de $\mathrm{Zn}$ y la continua aplicación de plaguicidas que contienen el microelemento, como causa de la toxicidad. Según resultados obtenidos por Casierra E Poveda (2005), alrededor de 350ppm de $Z n$ reducen el rendimiento y la calidad de fresa. El uso de lodos de albañal y aguas costeras contaminadas con Zn y metales pesados incrementó a niveles tóxicos su concentración en varios cultivos (Muchaweti et al. 2006)

Cloro: La toxicidad, se manifiesta en suelos salinos con exceso de cloruros, en suelos neutros o alcalinos, en suelos de zonas áridas o semiáridas o con influencia marina. Además, ocurre al emplear agua de riego rica en cloruros, más de $10 \mathrm{me} / \mathrm{L}$ y al fertilizar con cloruros de los cationes $\mathrm{K}, \mathrm{Ca}, \mathrm{Mg}, \mathrm{NH}_{4}{ }^{+}$y materiales ricos en cloruros (García, 1998; Lora, 1998; Tisdale et al. 1993; Marschner, 2003). 
Níquel: Brown et al. (1987) y Marschner (2003) mencionan, como condiciones de toxicidad, la aplicación de lodos y aguas de albañal y el bajo contenido de calcio y magnesio. En cebada, la fuente de níquel guardó relación con la fitotoxicidad y la concentración, cultivo en el cual Molas et al. (2004) encontraron que la absorción fue mayor a partir de $\mathrm{NiSO}_{4} 7 \mathrm{H}_{2} \mathrm{O}$ de citrato o de EDTA.

\section{Síntomas de toxicidad:}

A continuación, se anotan algunos síntomas generales de toxicidad para algunos de los micronutrimentos:

Manganeso: García (1998) y Marschner (2003) mencionan clorosis o necrosis marginal que aparece primero en las hojas más viejas, como en alfalfa, maní, trébol dulce; manchas intervenales pardas o café, rodeadas por una zona clorótica, como en cebada; hojas cloróticas con manchas necróticas y de tamaño reducido, como en tabaco y, hojas pequeñas y quebradizas, como en el algodonero. Estos autores demostraron, además, la pérdida de dominancia apical y proliferación de chupones axilares, como en el cacao y la aparición de síntomas de deficiencia de otros elementos, como $\mathrm{Ca}$, Fe y Mg. También mencionan problemas foliares, tales como hojas pequeñas, verde claras con bordes amarillentos en el cafeto, hojas nuevas con puntos pardos en las nervaduras finalmente amarillentas, como en soya y fríjol y hojas con manchas amarillentas entre las nervaduras, seguido de puntos pardos que se pueden juntar, como en el trigo. El efecto típico de la fitotoxicidad de Mn es una disminución exponencial en el crecimiento al incrementar la concentración de manganeso por encima del nivel crítico.

Cobre: Los síntomas de toxicidad por cobre, se manifiestan por hojas viejas con manchas acuosas y después pardas y negras, falta de crecimiento de hojas y muerte de raíces, como en el cafeto (INPOFOS, 1993); por la inhibición del crecimiento de la raíz y por la producción de síntomas de deficiencia de hierro (Marschner, 2003).

Zinc: La clorosis de las hojas nuevas, debido a deficiencia inducida de hierro es síntoma típico de toxicidad de Zn. Además en suelos de texturas gruesas, el exceso de zinc induce deficiencias de cobre en cebada, avena y trigo, principalmente (Lora, 1999; García, 1998).

Molibdeno: Marschner (2003), Motta (1976) e Higuita E Lora (1979) coinciden en mencionar como síntomas la deformación de las hojas y decoloración amarilla dorada en los brotes y una deficiencia de hierro.
Boro: Síntomas de toxicidad, se manifiestan por clorosis o necrosis o ambas en los márgenes y en los ápices de hojas maduras. En plantas monocotiledóneas, se presenta necrosis en los ápices y no en las márgenes, mientras que en las dicotiledóneas, se observa tanto necrosis marginal, como apical (Keren E Bingham, 1985; Marschner, 2003; IFDC, 1985).

Hierro: Para este micronutrimento, Escobar (1985), Bernal \& Gómez (2004) y Salisbury \& Ross (1994) indican, como síntomas, un anaranjamiento de las hojas de algunas variedades de arroz; eventualmente estas hojas se secan y la planta aparece como chamuscada (quemada); las panículas no se desarrollan y las raíces son pardas, gruesas y a veces espesas. Además, la toxicidad acentúa los síntomas de deficiencia de manganeso y de fósforo.

Cloro: Necrosis de los ápices de las hojas que progresan a lo largo de las márgenes y la caída temprana de las hojas cuando la toxicidad es muy elevada. Otros síntomas son parecidos a los ocasionados por problemas de salinidad (García, 1998; Marschner, 2003).

\section{EL ANÁLISIS FOLIAR}

Aunque el término indica análisis de "hojas", se refiere al análisis cuantitativo del contenido de un elemento en toda la parte aérea de la planta o en parte de ella. La parte comúnmente empleada para este análisis es la hoja o parte de ella. El análisis foliar permite el diagnóstico o confirmación de síntomas visuales, identificar la denominada "hambre escondida", indicar si los nutrientes aplicados vía foliar o edáfica han sido tomados, indicar interacciones o antagonismos entre nutrientes, evaluar el estado nutricional a través del tiempo y sugerir análisis o estudios adicionales para identificar problemas en una zona determinada (INPOFOS, 1993; Malavolta, 1994; Lora, 1998). El análisis foliar que envuelve a los elementos B, Cu, Fe, Mn, Zn y Mo, generalmente es más útil en cultivos perennes que en cultivos anuales.

Para que el análisis foliar sea realmente una herramienta útil, se deben tener en cuenta los siguientes aspectos:

Toma y preparación de muestras: Las muestras deben ser representativas del área y estar constituida por un número suficiente de submuestras, el cual puede ser calculado usando la fórmula de Peterson $\mathcal{E}$ Calvin (1965) que dice:

$$
N=T^{2} S^{2} \times 1 / D
$$


donde: $\mathrm{N}=$ No. de submuestras; $\mathrm{Ta}=\mathrm{T}$ de Student con (n-1) grados de libertad y "a" nivel de probabilidad; $\mathrm{S}^{2}=$ Varianza, y $\mathrm{D}=$ Límite específico de precisión.

El tipo de material, la edad más adecuada de la planta y el número de submuestras es muy importante. En la Tabla 1, se menciona información relevante para el muestreo en algunos cultivos (Malavolta, 1994; Howeler, 1985; Lora, 1970). En relación con la preparación de la muestra es conveniente tener en cuenta las siguientes consideraciones (Lora, 1998; Bornemiza, 1978): evitar pérdidas por respiración y descomposición (almacenar a $2-3^{\circ} \mathrm{C}$ ); emplear un adecuado cuarteo para muestras representativas, tanto antes como después de la molien- da; ejecutar una molienda adecuada $(0,4-0,8 \mathrm{~mm})$, en un molino de acero inoxidable; realizar un adecuado lavado, secado $\left(60-75^{\circ} \mathrm{C}\right.$ por $24-48$ horas), almacenamiento y evitar contaminación durante el proceso de análisis.

Análisis químico de las muestras: Existen varias metodologías. Para los elementos Fe, Cu, Mn y Zn, la digestión vía húmeda. Los nutrimentos se cuantifican por espectrofotometría de absorción atómica; para molibdeno, se incluye el horno de grafito. Para boro, se hace digestión seca, las cenizas se disuelven en ácido y el elemento se determina colorimétricamente (Lora, 1998; Vargas et al.2002; IFDC, 1985; Camelo et al. 1992).

Tabla 1. Muestreo para análisis foliar en algunos cultivos (Malavolta, 1994; Howeler, 1985; Lora, 1970).

\begin{tabular}{|c|l|l|c|}
\hline \multirow{2}{*}{ CULTIVO } & \multicolumn{1}{|c|}{ ÉPOCA } & \multicolumn{1}{|c|}{ PARTE DE LA PLANTA } & $\begin{array}{c}\text { NÚMERO DE } \\
\text { HOJAS/ha }\end{array}$ \\
\hline Arroz & Época media macollamiento & Lámina foliar hoja "Y" & $30-35$ \\
\hline Maíz & Iniciación barbas o cabello & Hoja opuesta a la mazorca principal & $30-35$ \\
\hline Fríjol & Inicio de la floración & Lámina foliar primera hoja madura & $30-35$ \\
\hline Papa & Inicio botón floral & Peciolo de la cuarta hoja a partir del cogollo & $30-45$ \\
\hline Yuca & Tres - cuatro meses de edad & Lámina foliar primera hoja completamente expandida & $30-35$ \\
\hline Palma Africana & Inicio estación seca preferencialmente & Folíolos medios de la hoja 17 o de la hoja 9 según la edad & $30-35$ \\
\hline Algodón & Inicio de la floración & Lámina de hojas en la axila de la "manzana" & $30-35$ \\
\hline Tomate de Mesa & Floración & Cuarta hoja a partir del cogollo & $40-45$ \\
\hline Leguminosas Forrajeras & Inmediatamente antes de la floración & Toda la parte aérea & $30-35$ \\
\hline
\end{tabular}

Nivel crítico: El nivel crítico de deficiencia es el contenido del elemento en la muestra por debajo del cual se espera una respuesta significativa a la aplicación del elemento. El nivel crítico de toxicidad hace referencia al contenido del elemento por encima del cual la planta sufre por toxicidad del elemento y, por tanto, hay reducción en el rendimiento. En general, el nivel adecuado o suficiente está entre el nivel de deficiencia y de toxicidad. En la Tabla 2 aparecen las concentraciones de micronutrimentos en algunos cultivos (Howeler, 1985; Malavolta, 1994) para tres niveles. Esta información es a nivel general, ya que en muchos casos puede haber diferencia entre variedades e híbridos dentro de la misma especie; por este motivo, se requiere determinar los niveles críticos de toxicidad por zonas y por cultivos. Por ejemplo, para maíz, niveles en hojas de boro, manganeso, hierro, cobre y zinc superiores a 35, 200, 350, 50 y 100ppm, respectivamente, se han determinado en suelos localizados en zona tropical con temperaturas elevadas y precipitaciones frecuentes (Howeler, 1985). Al presentar el cultivo presenta exceso de micronutrientes, el desarrollo, en algunos casos, se restringe y los rendimientos se pueden reducir significativamente. El manejo de los micronutrimentos debe ser cuidadoso para evitar problemas de toxicidad. En muchos casos es más grave la toxicidad que la deficiencia.

\section{EL ANÁLISIS DE SUELOS}

El análisis de suelos tiene como propósito caracterizar el recurso suelo para tomar decisiones sobre el uso de fertilizantes y enmiendas y conocer si existe deficiencia o toxicidad de algunos elementos para así hacer el manejo 
Tabla 2. Concentración de micronutrientes para los niveles deficiente, normal y tóxico para algunos cultivos (Howeler, 1985).

\begin{tabular}{|c|c|c|c|c|c|c|c|c|}
\hline \multirow{2}{*}{ CULTIVO } & \multirow{2}{*}{ PARTE DE LA PLANTA } & \multirow{2}{*}{ EPOCA } & \multirow{2}{*}{$\begin{array}{c}\text { ESTADO } \\
\text { NUTRICIONAL* }\end{array}$} & \multicolumn{5}{|c|}{ ppm } \\
\hline & & & & B & Mn & $\mathrm{Fe}$ & $\mathrm{Cu}$ & $\mathrm{Zn}$ \\
\hline \multirow[t]{3}{*}{ Maíz } & \multirow[t]{3}{*}{ Hoja opuesta a la mazorca principal } & \multirow[t]{3}{*}{ Iniciación cabello } & $D<$ & 2 & 15 & 10 & 2 & 10 \\
\hline & & & $\mathrm{N}$ & 10 & 85 & 135 & 13 & 45 \\
\hline & & & $T>$ & 35 & 200 & 350 & 50 & 100 \\
\hline \multirow[t]{3}{*}{ Arroz } & \multirow[t]{3}{*}{ Lámina foliar hoja "Y" } & \multirow[t]{3}{*}{ Mitad macollamiento } & $D<$ & 3 & 20 & 70 & 6 & 10 \\
\hline & & & N & 50 & 120 & 250 & 15 & 35 \\
\hline & & & $T>$ & 100 & 2500 & 300 & 30 & 1500 \\
\hline \multirow[t]{3}{*}{ Frijol } & \multirow[t]{3}{*}{ Lámina foliar primera hoja madura } & \multirow[t]{3}{*}{ Inicio floración } & $D<$ & 15 & 20 & 100 & 15 & 15 \\
\hline & & & $\mathrm{N}$ & 25 & 140 & 400 & 20 & 45 \\
\hline & & & $T>$ & 45 & 200 & 700 & 30 & 90 \\
\hline \multirow[t]{3}{*}{ Yuca } & \multirow[t]{3}{*}{ Lámina foliar primera hoja expandida } & \multirow[t]{3}{*}{ Tres - cuatro meses de edad } & $D<$ & 20 & 45 & 100 & 5 & 25 \\
\hline & & & $\mathrm{N}$ & 45 & 80 & 130 & 8 & 20 \\
\hline & & & $T>$ & 100 & 250 & 200 & 15 & 120 \\
\hline
\end{tabular}

$\mathrm{D}=$ Deficiente $\mathrm{N}=$ Normal $\mathrm{T}=$ Tóxico

pertinente (Lora, 1998). Los objetivos del análisis de elementos menores del suelo son separar áreas deficientes de áreas no deficientes, determinar la probabilidad de respuesta rentable a la aplicación de micronutrimentos, tanto por rendimiento como por calidad, evaluar la productividad del suelo y determinar las condiciones del suelo que pueden ser mejoradas mediante prácticas de cultivo y adición de enmiendas.

Toma de muestras: Dada la heterogeneidad de factores de suelo que afectan la disponibilidad de micronutrimentos es recomendable tomar suficiente número de submuestras para constituir una muestra. Un estimativo de las submuestras a tomar, se puede derivar de la fórmula propuesta por Peterson E Calvin (1965), tal como se explicó para el análisis foliar. La profundidad de muestreo generalmente se recomienda a $20 \mathrm{~cm}$, pero se puede llegar hasta $40 \mathrm{~cm}$ dependiendo del cultivo. Para evitar contaminaciones no se debe emplear hierro galvanizado, bronce, latón o caucho en el equipo de muestreo o en los recipientes donde se coloca la muestra. El acero inoxidable o algunos tipos de plástico pueden ser recomendables.

Determinación de los micronutrimentos: De acuerdo con Lora (1998) las bases químicas para el análisis de los micronutrimientos corresponden a los factores de intensidad y de capacidad, que afectan la disponibilidad del nutrimento para la planta y, por tanto, deben ser reflejados en un análisis de suelos: a los extractantes ácidos para suelos ácidos, a los extractantes básicos para suelos neutros o alcalinos y a agentes quelatantes y complejantes para micronutrimentos catiónicos. Debido a que la cantidad de micronutrimentos intercambiables es muy baja, muchos extractantes contienen ácidos, bases, agentes complejantes, que disuelven también otras formas de nutrimento y así se tiene una medida adecuada de la capacidad del suelo para suministrar los micronutrimentos a la planta. Importante tener en cuenta algunas consideraciones relacionadas con los extractantes y las extracciones simultáneas, como acetato de amonio - EDTA para Fe, Mn, Zn, Cu, Mo, Co; DTPA para Zn, Fe, $\mathrm{Mn}, \mathrm{Cu}$; bicarbonato de sodio para $\mathrm{Fe}, \mathrm{Cu}, \mathrm{Mn}, \mathrm{Zn}, \mathrm{y}$ bicarbonato de sodio más DTPA para microcationes.

Nivel crítico: El nivel crítico hace referencia al contenido o rango por debajo del cual la probabilidad de obtener bajos rendimientos es elevada y por encima la respuesta a la aplicación del elemento es adecuada. Existe un nivel crítico de toxicidad por encima del cual hay disminución en calidad y en rendimiento. Para que los análisis tengan utilidad es necesario hacer la calibración de los métodos 
analíticos, para lo cual, preferencialmente, se deben emplear los estudios de campo donde se correlaciona el rendimiento con la cantidad extraída y determinada, o en su defecto la cantidad o la concentración foliar con lo extraído del suelo por la solución extractora bajo estudio. En la Tabla 3 aparecen los niveles críticos para algunos cultivos de acuerdo al extractante utilizado en la determinación de los micronutrimentos.

Tabla 3. Niveles críticos de elementos menores en el suelo (Lora, 1998).

\begin{tabular}{|c|c|c|c|}
\hline ELEMENTO & EXTRACTANTE & NIVEL (PPM)* & CULTIVO \\
\hline \multirow[t]{4}{*}{ Boro } & Agua Caliente & $\begin{array}{l}\mathrm{B}<0,6 \\
\mathrm{~T}>0,8\end{array}$ & Papa \\
\hline & & $\begin{array}{l}\mathrm{B}<0,2 \\
\mathrm{~T}>0,4\end{array}$ & Hortalizas \\
\hline & $\mathrm{Ca}\left(\mathrm{H}_{2} \mathrm{PO}_{4}\right)_{2} 0,08 \mathrm{M}$ & $\begin{array}{l}\mathrm{B}<0,4 \\
\mathrm{~T}>0,6\end{array}$ & Papa \\
\hline & & $\begin{array}{l}B<0,25 \\
T>0,40\end{array}$ & Sorgo \\
\hline \multirow[t]{2}{*}{ Cobre } & $\mathrm{NaHCO}_{3} 0,5 \mathrm{~N}+$ EDTA $0.05 \mathrm{M}(\mathrm{pH} 8,5)$ & $\begin{array}{l}B<1,0 \\
T>2,0\end{array}$ & General \\
\hline & $\mathrm{HCl} 0,05 \mathrm{~N}+\mathrm{H}_{2} \mathrm{SO}_{4} 0,025 \mathrm{~N}$ & $\begin{array}{l}\mathrm{B}<0,5 \\
\mathrm{~T}>1,0\end{array}$ & Pastos \\
\hline \multirow[t]{2}{*}{ Manganeso } & $\mathrm{NaHCO}_{3} 0,5 \mathrm{~N}+$ EDTA $0.05 \mathrm{M}(\mathrm{pH} 8.5)$ & $\begin{array}{l}\mathrm{B}<5,0 \\
\mathrm{~T}>8,0\end{array}$ & General \\
\hline & $\mathrm{HCl} 0,5 \mathrm{~N}+\mathrm{H}_{2} \mathrm{SO}_{4} 0,025 \mathrm{~N}$ & $\begin{array}{l}\mathrm{B}<5,0 \\
\mathrm{~T}>8,0\end{array}$ & Frijol y Soya \\
\hline \multirow[t]{2}{*}{ Hierro } & $\mathrm{NaHCO}_{3} 0,5 \mathrm{~N}+\mathrm{EDTA} 0,05 \mathrm{M}(\mathrm{pH} 8,5)$ & $\begin{array}{l}\mathrm{B}<25 \\
\mathrm{~T}>50\end{array}$ & General \\
\hline & $\mathrm{HCl} 0,1 \mathrm{~N}$ & $\begin{array}{l}\mathrm{B}<0,5 \\
\mathrm{~T}>1,5\end{array}$ & $\begin{array}{c}\text { Maíz } \\
\text { Hortalizas }\end{array}$ \\
\hline \multirow[t]{2}{*}{ Zinc } & $\mathrm{NaHCO}_{3} 0,5 \mathrm{~N}+\mathrm{EDTA} 0.1 \mathrm{M}(\mathrm{pH} 8,5)$ & $\begin{array}{l}B<1,5 \\
T>3,0\end{array}$ & General \\
\hline & $\mathrm{HCl} 0,05 \mathrm{~N}+\mathrm{H}_{2} \mathrm{SO}_{4} 0,025 \mathrm{~N}$ & $\begin{array}{l}\mathrm{B}<0,5 \\
\mathrm{~T}>1,0 \\
\mathrm{~B}<0,8 \\
\mathrm{~T}>1,5\end{array}$ & $\begin{array}{l}\text { Pastos } \\
\text { Soya y Frijol }\end{array}$ \\
\hline Molibdeno & Oxala to ácido de amonio ( $\mathrm{pH} 3,3)$ & $\begin{array}{l}B<0,10 \\
T>0,20\end{array}$ & General \\
\hline \multirow[t]{2}{*}{ Cloro } & Extracto de saturación & $\begin{array}{l}B<5 \mathrm{me} / \mathrm{L} \\
T>6 \mathrm{me} / \mathrm{L}\end{array}$ & $\begin{array}{c}\text { Aguacate, tabaco, } \\
\text { fresa }\end{array}$ \\
\hline & & $\begin{array}{l}\mathrm{B}<15 \mathrm{me} / \mathrm{L} \\
\mathrm{T}>20 \mathrm{me} / \mathrm{L}\end{array}$ & $\begin{array}{l}\text { Cíticos, uva, } \\
\text { frutales de hueso }\end{array}$ \\
\hline
\end{tabular}




\section{ELEMENTOS TRAZA}

Dentro de los elementos traza algunos son esenciales para la planta, como lo es el caso de los micronutrimentos. Algunos son tóxicos si se acumulan en el suelo o en los tejidos foliares, como ocurre con el cadmio, el mercurio, el plomo, el arsénico, el cromo, el flúor, el vanadio y el selenio. El empleo de aguas negras y agua de riego de mala calidad puede conllevar cantidades tóxicas de elementos traza a suelos y plantas y, por tanto, a animales y el hombre. En la Tabla 4, se presentan las concentraciones permisibles de elementos traza en aguas de riego (García, 1998; Alfaro et al. 2002; Matamoros, 2003; Matamoros et al. 1999).

Tabla 4. Máximas concentraciones permisibles de elementos traza en el agua de riego (García, 1998; Matamoros, 2003)).

\begin{tabular}{|c|c|c|c|}
\hline ELEMENTO & $\begin{array}{c}\text { MÁXIMA } \\
\text { CONCENTRACIÓN } \\
(\mathbf{m g} / \mathbf{L})\end{array}$ & ELEMENTO & $\begin{array}{c}\text { MÁXIMA } \\
\text { CONCENTRACIÓN } \\
\text { (mg/L) }\end{array}$ \\
\hline Arsénico (As) & 0,10 & Manganeso (Mn) & 0,20 \\
\hline Cadmio (Cd) & 0,01 & Molibdeno (Mo) & 0,01 \\
\hline Cromo (Cr) & 0,10 & Níquel (Ni) & 0,20 \\
\hline Cobre (Cu) & 0,20 & Plomo (Pb) & 5,0 \\
\hline Flúor (F) & 1,0 & Selenio (Se) & 0,02 \\
\hline Hierro (Fe) & 5,0 & Zinc (Zn) & 2,0 \\
\hline Litio (Li) & 2,5 & & \\
\hline
\end{tabular}

\section{POSIBLES SOLUCIONES ALA CONTAMINACIÓN POR MICROELEMENTOS}

En general es más difícil recuperar un suelo que presenta exceso de micronutrimentos en forma disponible, que cuando hay deficiencia. Por este motivo es importante conocer el suelo y el cultivo, para llevar a cabo prácticas preventivas que reduzcan la posibilidad de toxicidad. Entre éstas están las siguientes: caracterizar el estado de disponibilidad del elemento para aplicar solo la cantidad requerida de acuerdo al cultivo; emplear aguas de riego de buena calidad que tengan niveles bajos de elementos menores; no utilizar materiales ricos en elementos menores, como aguas negras, lodos de albañal, aguas ricas en B, Se o Mo; no emplear fertilizantes orgánicos ricos en elementos menores, especialmente si la dosis a aplicar es elevada. Suelos regados con agua del río Bogotá en su cuenca alta han mostrado contenidos elevados de $\mathrm{Pb}, \mathrm{Ni}, \mathrm{Cr}$, Co y vanadio, potencialmente tóxicos para la salud pública y animal al ingerir productos agrícolas que han crecido en estos suelos (Matamoros et al. 1999). La contaminación por exceso de $\mathrm{Cr}$ procedente de las curtimbres puede ser tóxica para hombres y animales que consuman el material vegetal contaminado. La elevación del pH o la aplicación de yeso puede reducir el problema (Álvarez et al. 2006).

El Se es un elemento esencial para los animales pero no para las plantas. La máxima cantidad de Se en el agua para riego es de 0,020ppm; para el consumo humano y animal, se recomienda solo hasta 0,01ppm (Lora et al. 1998). En Colombia, se presentan problemas de aguas altas en Se que deben ser tratadas para reducir la concentración del elemento hasta niveles no tóxicos, por medio de un procedimiento que incluye la reducción de los selenatos a selenitos y arrastre de éstos con gel de hidróxido férrico; el contenido de selenio se elimina a niveles no tóxicos, esto es menores de 0,007ppm.

El Se y el Mo, por encima de ciertos niveles, son tóxicos y pueden producir seleniosis y molibdenosis en animales y en humanos que ingieran productos vegetales ricos en estos elementos. En el país existe el problema en suelos influenciados por sedimentos del Cretáceo (formación Villeta o equivalentes) (García et al. 1999; Mejía E Oso- 
rio, 1983; Puentes E Rodríguez, 1983). Para reducir el problema, se ha investigado con el empleo de sulfato de calcio, cloruro de calcio y sulfato de amonio. En suelos procedentes del municipio de Sutamarchán, se redujo la concentración de molibdeno en avena variedad ICA-Cajicá de 32ppm a 21ppm, con la aplicación de $1000 \mathrm{~kg} / \mathrm{ha}$ de cualquiera de los materiales sulfato de calcio, cloruro de calcio o sulfato de amonio (Puentes E Rodríguez, 1983).

Por su parte, en suelos seleníferos del municipio de Puerto Boyacá, a orillas del río Negro y derivados de la Formación Villeta, se determinó el efecto de dosis variables de cloruro de calcio, sulfato de calcio, cloruro de bario y sulfato de bario sobre la concentración de selenio en el pasto Ryegrass (Lolium multiflorum). Los resultados muestran que la aplicación de $500-750 \mathrm{~kg} / \mathrm{ha}$ de cualquiera de los materiales redujo la concentración de selenio en el pasto a niveles inocuos para animales y humanos (Torres et al. 1978). Por ser de un costo más bajo y por la facilidad de adquisición en el mercado, las sales de calcio pueden ser muy promisorias para el manejo del problema.
En suelos seleníferos procedentes de Útica (Cundinamarca), se determinó el efecto en la reducción de la absorción de selenio por dosis variables de sulfato de calcio y cloruro de calcio, por parte de las especies maíz híbrido ICA-H-211, fríjol variedad Diacol Calima, avena variedad ICA-Cajicá y pasto Angleton. Las dosis empleadas de cada uno de los compuestos fueron 0 , 400, 800, 1200, 1600kg/ha. En general, se observó efecto inhibitorio de la absorción de selenio en las cinco especies (Mejía \& Osorio, 1983), tal como se ve en la Tabla 5. Empleando el mismo suelo utilizado en este estudio, se evaluó, bajo condiciones de casa de malla, el efecto de las mismas fuentes y dosis variables sobre la concentración de selenio en la espiga de trigo variedad Sugamuxi. Se inhibió la concentración de selenio a niveles inferiores a 5ppm, consideradas no tóxicas con el empleo de sulfato de amonio y sulfato de calcio, debido posiblemente al bloqueo en la absorción de selenio por el trigo a causa de las emiendas aplicadas.

El pH es uno de los factores que tiene más efecto en la disponibilidad para la planta de los elementos menores y pesados. Sin embargo, el único elemento menor cuya

Tabla 5. Efecto de dosis y fuentes de calcio y sulfato de amonio en la concentración de selenio en cuatro especies vegetales (Mejía E Osorio, 1983).

\begin{tabular}{|c|c|c|c|c|c|}
\hline \multirow{2}{*}{ Fuentes } & \multicolumn{5}{|c|}{$\mathrm{Se}(\mathrm{ppm})$} \\
\hline & Dosis & Maíz & Fríjol & Avena & Angleton \\
\hline$\left(\mathrm{NH}_{4}\right)_{2} \mathrm{SO}_{4}$ & $\begin{array}{c}0 \\
400 \\
800 \\
1200 \\
1600\end{array}$ & $\begin{array}{c}19,50 \\
16,00 \\
11,00 \\
4,10 \\
3,70\end{array}$ & $\begin{array}{c}21,10 \\
16,50 \\
13,90 \\
5,40 \\
3,15\end{array}$ & $\begin{array}{c}18,70 \\
14,00 \\
13,00 \\
9,80 \\
10,00\end{array}$ & $\begin{array}{c}18,80 \\
12,40 \\
9,60 \\
8,00 \\
9,00\end{array}$ \\
\hline $\mathrm{CaSO}_{4}$ & $\begin{array}{c}0 \\
400 \\
800 \\
1200 \\
1600\end{array}$ & $\begin{array}{c}19,50 \\
10,50 \\
10,20 \\
8,50 \\
5,70\end{array}$ & $\begin{array}{c}21,10 \\
21,00 \\
15,80 \\
5,40 \\
5,20\end{array}$ & $\begin{array}{c}18,70 \\
12,00 \\
9,30 \\
9,70 \\
9,40\end{array}$ & $\begin{array}{c}18,80 \\
12,00 \\
11,30 \\
8,30 \\
8,00\end{array}$ \\
\hline $\mathrm{CaCl}_{2}$ & $\begin{array}{c}0 \\
400 \\
800 \\
1200 \\
1600\end{array}$ & $\begin{array}{c}19,50 \\
14,20 \\
11,00 \\
3,30 \\
3,70\end{array}$ & $\begin{array}{c}21,10 \\
15,70 \\
14,40 \\
11,10 \\
4,90\end{array}$ & $\begin{array}{c}18,70 \\
11,70 \\
8,70 \\
6,00 \\
6,70\end{array}$ & $\begin{array}{c}18,80 \\
14,90 \\
13,60 \\
7,00 \\
7,00\end{array}$ \\
\hline
\end{tabular}


disponibilidad para la planta se incrementa al elevar el $\mathrm{pH}$ es el Mo. Bajo este contexto puede ser recomendable lo expresado a continuación para controlar o, por lo menos reducir, el exceso de contenido disponible de los micronutrimentos (Lora, 1994; Brown et al. 1972; García, 1998; Bohn et al. 2001; Gyana, 2003; Lora, 2001).

Boro: Aplicación de cal hasta pH de 6,50; incrementar el nivel de Ca y K.

Molibdeno: Reducir el pH a niveles de 4,5-5,0 o usar fertilizantes amoniacales o azufre elemental pulverizado; aplicación de yeso o de sulfato de amonio (efecto del azufre).

Hierro : Encalamiento a pH de 7,0; fertilización con potasio; empleo de variedades tolerantes del cultivo; fertilización fosfórica elevada.

Manganeso: Encalamiento a pH de 6,0-6,5; aplicación elevada de magnesio; aplicación elevada de fósforo; empleo de genotipos tolerantes; aplicación elevada de materia orgánica.

Cobre: Encalamiento a pH de 6,5-7,0; incrementar el nivel de nitrógeno; incorporación de materia orgánica. Zinc: Incrementar el pH por encima de 6,5 por encalamiento; complejamiento con ácidos húmicos y fúlvicos; fertilización fosfórica elevada.

Níquel y Cromo: Especialmente en suelos influenciados por serpentina puede ser adecuado el encalamiento hasta $\mathrm{pH}$ alrededor de 6 a 7 (Marschner, 2003).

Cadmio y Plomo: El encalamiento o la aplicación de tierra diatomácea activada puede reducir la toxicidad de estos elementos (Méndez et al. 2000). Por su parte, la aplicación de sulfato de hierro o sulfato de aluminio puede reducir la toxicidad de arsénico (Liebig, 1966). La fitorremediación con plantas con buena tolerancia al exceso de metales pesados puede ser una opción para suelos contaminados. Zhelgazcov et al. (2006) encontraron, por ejemplo, que la composición y presencia de $\mathrm{Cd}, \mathrm{Pb}$, y $\mathrm{Cu}$ no fue significativo para el contenido de aceites esenciales de menta, eneldo y basilico.

Selenio: El sulfato de amonio o el sulfato de Ca reducen la concentración del elemento hasta niveles no tóxicos en varios cultivos (Mejía \& Osorio, 1983).
Finalmente, suelos sulfatados con exceso de Mn, Fe y $S$ pueden ser recuperados por encalamiento y posterior lavado con drenajes adecuados y utilizando avena como cultivo; después de la avena se puede utilizar el terreno para siembras de algunas hortalizas y pastos (Gómez et al. 2005).

\section{BIBLIOGRAFÍA}

ABBAS, S.; SARFAZ, M.; MEHDI, S.; HASSAN, G.; REDHMMAN, O. 2006. Trace elements accumulation in soil and rice plants irrigated with contaminated water. Soil Tillage Res. (in press).

ACOSTA, A. 2003. Evaluación de la disponibilidad de elementos menores en algunos suelos de la serie Río Bogotá. Trabajo de Grado. Ing. Agrónomo. Universidad de Ciencias Aplicadas y Ambientales. Bogotá, D.C. 140p.

AGREDA, L.; OTERO, D.; ORTEGA, J. 1992. Disponibilidad de micronutrimentos bajo bosque y pradera en suelos de la llanura del Pacífico, Dept. de Nariño. Suelos Ecuatoriales. 12(1):14-23.

ALFARO, R.; GARCÍA, E.; MONTENEGRO, O. 2002. Niveles de contaminación de mercurio, cadmio, arsénico y plomo en subsistemas de producción de la cuenca baja del Río Bogotá. Rev. U.D.C.A Act. \& Div. Cient. 4(2):66-71.

ÁLVAREZ, D.; CONTRERAS, S.; TRUJILLO, N.; FRÍAS, F.; OLALDE, V. 2006. Effects of tanneries on chemical and biological soil characteristics. Appl. Soil Ecol. 33:269-277.

BARRERA, L. 2003. El papel de la materia orgánica en el manejo integral de la fertilidad del suelo. En: Soc. Col. de la Ciencia del Suelo. Manejo integrado de la fertilidad del suelo. Edit. Guadalupe Ltda. (Bogotá). p.123-134.

BERNAL, C.; TRIANA, P. 1980. Disponibilidad de micronutrientes en cuatro suelos de clima frío de Cundinamarca y Boyacá. Tesis Químico. U.N. de Colombia, Bogotá. 260p.

BERNAL, H.; GÓMEZ, R. 2004. Comportamiento de la papa criolla (Solanum phureja) a la aplicación de $\mathrm{Fe}, \mathrm{Cu}, \mathrm{Zn}, \mathrm{B}$ y Mo en suelos de la serie Bermeo. 
Trabajo de Grado Ing. Agrónomo. Universidad de Ciencias Aplicadas y Ambientales. Bogotá. $117 p$.

BOHN, H.; McNEAL, B.; O'CONNOR, G. 2001. Soil Chemistry. $3^{\text {rd }}$ Ed. John Wiley and Sons Inc. N.Y. 307p.

BORNEMIZA, E. 1978. Metodología para la investigación de problemas de micronutrientes. Suelos Ecuatoriales. 9(2):148-154.

BROWN, J.; AMBLER, E.; CHANLEY, L.; FOY, C. 1972. Different responses of plant genotypes to micronutrients . En: Dinauer, R. Micronutrients in Agriculture. Soil Sci. Soc. of Am. Inc., Madison, Wisconsin. p.389-418.

BROWN, P.; WELCH, M.; CARY, E. 1987. Nickel: a micronutrient essential for higher plants. Plant Physiol. 85:801-803.

BURBANO, H. 1998. Interacciones de la materia orgánica y los elementos menores. En: Memorias Curso Actualidad y futuro de los micronutrimentos en la agricultura. Soc. Col. de la Ciencia del Suelo. Prolabo Ltda. Santa Fe de Bogotá, D.C. p.31-47.

CAMELO, D.; ROJAS, A.; YUNDA, A. 1992. Evaluación de métodos para la extracción de boro disponible en suelos. Suelos Ecuatoriales. 12(1):310-327.

CASIERRA, F.; POVEDA. J. 2005. La toxicidad por Mn y $\mathrm{Zn}$ disminuye la calidad y rendimiento en fresa. Agron. Colomb. 23(2):283-289.

CASTILLO, E. 1985. Disponibilidad de micronutrientes en cinco suelos de la cuenca media del río Suárez (Santander y Boyacá). Tesis M.Sc. Progr. de Estudios para Graduados ICA-UN. Bogotá. 188p.

DAI, J.; BECQUER, T.; ROUILLER, J.; REVERSAT, F.; LAVELLE, P. 2004. Influence of heavy metals on $\mathrm{C}$ and $\mathrm{N}$ mineralization and microbial biomass in $\mathrm{Zn}, \mathrm{Pb}, \mathrm{Cu}$ and $\mathrm{Cd}$ contaminated soils. Appl. Soil Ecol. 95:99-109.

ESCOBAR, R. 1985. Disponibilidad de micronutrimentos en suelos de los Llanos Orientales. Tesis M.Sc.
Progr. de Estudios para Graduados ICA-UN. Bogotá. 113p.

FASSBENDER, H. 1982. Química de suelo con énfasis en suelos de América Latina. Elementos menores. Inst. Interam. de Cooperación para la Agricultura. San José de Costa Rica. p.362-390.

FREY, B.; STEMMER, M.; WIDMER,F.; LUSTER, J.; SPERISEN, Ch. 2006. Microbial activity and comunity structure of a soil after heavy metal contamination in a model forest ecosystem. Soil Biol. Bioquem. 38:1745-1756.

GARAVITO, F.; LEÓN, A. 1978. Propiedades del suelo en relación con deficiencias de boro en el Valle del Cauca. Suelos Ecuatoriales. 9(2):105-201.

GARCÍA, B. 1981. Disponibilidad de elementos menores en cuatro suelos del municipio de Villeta. Tesis M.Sc. Progr. de Estudios para Graduados ICA-UN. Bogotá. 132p.

GARCÍA, A. 1998. Efectos tóxicos de los micronutrientes y otros elementos. En: Memorias Curso Actualidad y futuro de los micronutrimentos en la agricultura. Soc. Col. de la Ciencia del Suelo. Santa Fe de Bogotá, D.C. p.13-31.

GARCÍA, A. 1994. Diagnóstico y control de la fertilidad en suelos afectados por sales y sodio. En: Soc. Col. de la Ciencia del Suelo. Fertilidad de suelo, diagnóstico y control. Edit. Guadalupe Ltda.Bogotá, D.C. p.469-506.

GARCÍA, C.; CABRERA, D.; MEJÍA, L.; ROJAS, A. 1999. Evaluación del contenido de selenio y molibdeno en suelos y pastos de dos fincas ganaderas de Puerto Salgar (Cundinamarca) y sus posibles efectos en la población pecuaria. Suelos Ecuatoriales. 29(2):150-155.

GÓMEZ, I.; CASTRO, H.; PACHECO, W. 2005. Recovery and management of actual acid and sulphate soils in Boyacá, Colombia. Agron. Colomb. 23(1):128135.

GRANDETT, G. 1979. Disponibilidad de manganeso en cuatro series de suelos de la Sabana de Bogotá. 
Tesis M.Sc. Progr. de Estudios para Graduados ICA-UN. Bogotá. 115p.

GUERRERO, R.; BURBANO, H. 1979. Fracciones de azufre y niveles críticos pa la planta en suelos de los Llanos Orientales y la Sabana de Bogotá. Suelos Ecuatoriales. 10:232-244.

GUTIÉRREZ. D. 1974. Fertilización del maíz H-302 confósforo y zinc en el oriente de Cundinamarca. Tesis M.Sc. Progr. de Estudios para Graduados ICA-UN. Bogotá. 107p.

GYANA, R. 2003. Effect of metal toxicity on plant growth and metabolism: I- Zinc. Agronomie (Francia). 23:3-11.

HIGUITA, F.; LORA, R. 1979. Respuesta de la coliflor a la aplicación de cal y molibdeno. Agric. Trop. (Colombia). 20(11):638.

HOWELER, R. 1985. Análisis foliar en el diagnóstico de problemas nutricionales. En: Curso Suelos y fertilización de cultivos. Soc. Col. de la Ciencia del Suelo. Neiva. p.37-61.

INTERNATIONAL FERTILIZER DEVELOPMENT CENTER IFDC. 1985. MIcronutrients in tropical food crop production. Ed. Vleck, P. Muscle Schools, Alabama (EEUU). 260p.

INSTITUTO DE LA POTASA Y EL FÓSFORO INPOFOS. 1993. Diagnóstico del estado nutricional de los cultivos. Quito. Ecuador. p.55.

JIMÉNEZ, J. 1983. Disponibilidad de cobre, manganeso y molibdeno en el Valle de Ubaté-Chiquinquirá y su relación con los nitratos en la planta. Tesis M.Sc. Progr. de Estudios para Graduados ICA-UN. Bogotá. 74p.

KEREN, R.; BINGHAM, J. 1985. Boron in water, soils and plants. En: Advances in Soil Science. Vol. 1. Edit. Springer Verlag N.Y. p.229-226.

KIRILKAYA, R.; AZKIN,T.; BAYRAKLI, B.; SAGLAN, M. 2004. Microbiological characteristics of soils contaminated with heavy metals. Europ. J. Soil Biol. 40:95-102.
LIEBIG, G. 1966. Arsenic. En: University of California. Diagnostic criteria for plants and soils. Edit. Chapman H. p.13-23.

LINDSAY, W. 1973. Inorganic phase eqilibrio of micronutrients in soils. Micronutrients in Agricultura. SSSA, Madison, Wisconsin. p.41-57.

LORA, R. 1970. Análisis foliar. Técnicas de muestreo y preparación de material para análisis químico. Instituto Colombiano Agropecuario ICA, Bogotá. 24p.

LORA, R. 1978. Respuesta de los cultivos de clima frío a la aplicación de micronutrientes. En: Ed. Silva, F. Memorias del V Eloquio de suelos. Potasio y micronutrientes en la agricultura colombina. Suelos Ecuatoriales. 9(2):183-191.

LORA, R. 1989. Factores que afectan la disponibilidad de azufre y elementos menores. En: Memorias Curso de actualización sobre suelos y fertilizantes. Instituto Colombiano Agropecuario ICA. C.I. Tibaitatá. Bogotá. p.129-153.

LORA, R. 1994. Factores que afectan la disponibilidad de nutrimentos para las plantas. En: Soc. Col. de la Ciencia del Suelo. Fertilidad de Suelos. Diagnóstico y Control. Santa Fe de Bogotá, D.C. p.29-57.

LORA, R. 1998. Análisis de suelos y material vegetal para micronutrimentos. En: Soc. Col. de la Ciencia del Suelo. Actualidad y futuro de los micronutrimentos en la agricultura. Edit. Prolabo Ltda. Santa Fe de Bogotá, D.C. p.47-56.

LORA, R.; MELO, T.; RODRÍGUEZ, J. 1998. Recuperación de aguas seleniferas. Rev. U.D.C.A Act. \& Div. Cient. (Bogotá) 1(2):77-85.

LORA, R. 1999. Dinámica del azufre y los micronutrimentos. En: Ciclo de cursos de actualización de conocimientos con aplicación en palma africana de aceite. CENIPALMA. Santa Fe de Bogotá, D.C. p.93-109.

LORA, R. 2001. El silicio en la agricultura, con énfasis en la producción de arroz. En: Soc. Col. de la Ciencia 
del Suelo. Los elementos secundarios y el silicio en la agricultura. p.125-135.

LORA, R.; ÁVILA, O.; RODRÍGUEZ, A. 2002a. Respuesta de la papa criolla a fuentes y dosis de boro en un Andisol de Cundinamarca. Suelos Ecuatoriales. 32:23-28.

LORA, R.; RAMÍREZ, J.; OLIVAR, A.; CABEZAS, M. 2002b. Respuesta de tres variedades de arroz a la aplicación de zinc en Villanueva (Casanare). Suelos Ecuatoriales. 32:1-6.

LORA, R. 2003. Las propiedades químicas del suelo y su fertilidad. En: Soc. Col. de la Ciencia del suelo. Manejo integral de la fertilidad del suelo. Edit. Guadalupe. Bogotá. p.31-42.

LORA, R. 2005. Efecto residual de la fertilización. En: Memorias Primer taller nacional sobre suelos. Fisiología y nutrición en papa. Cevipapa. Bogotá. p.83-86.

LORA, R.; TORRES, J.; BOLAÑOS, R. 2004. Disponibilidad de micronutrimentos en cuatro localidades de la serie Bermeo. Rev. U.D.C.A Act. \& Div. Cient. 7(1):91-100.

LORA, R.; MONTAÑEZ, I.; BERNAL, H.; GÓMEZ, R. 2006. Evaluación de la aplicación de $\mathrm{Fe}, \mathrm{Cu}, \mathrm{Mn}$, Zn, B y Mo en papa criolla en un Andisol de Cundinamarca. Suelos Ecuatoriales. 36:1-5.

LUCHO, C.; PRIETO, F.; DEL ROZO, M.; RODIRÍGUEZ, R.; PAGGI, H. 2005. Chemical fractionation of boron and heavy metals in soils irrigated with wastewater in central Mexico. Agric. Ecos. Environm. 108:57-71.

LUJÁN, M. 2005. El intrigante cadmio. Cátedra de química biológica vegetal. Universidad de Buenos Aires. 5p.

MALAVOLTA, E. 1994. Diagnóstico Foliar. En: Soc. Col. de la Ciencia del Suelo. Fertilidad de Suelos. Diagnóstico y Control. Santa Fe de Bogotá. p.57-99.

MARIN, G. 1986. Fertilidad de suelos con énfasis en Colombia. Instituto Agropecuario de Colombia ICA, Bogotá. 193p.
MARSCHNER, H. 2003. Mineral nutrition of higher plants. Function of nutrients: micronutrients. Diagnosis of deficiency and toxicity of mineral nutrients. Edit. Acad. Press. San Diego, California (EEUU). p.313-404 y 461-469.

MATAMOROS, A.; VARGAS, O.; MEJÍA, L. 1999. Especiación química del $\mathrm{Pb}, \mathrm{Ni}, \mathrm{Cr}, \mathrm{Cu}, \mathrm{Cd}$ y $\mathrm{V}$ en dos suelos naturales y en un Andosol agrícolas, contaminados por riego con las aguas del río Bogotá en su cuenca alta. Suelos Ecuatoriales. 29(2):143-185.

MATAMOROS, A. 2003. Suelos contaminados por metales traza. En: Soc. Col. de la Ciencia del Suelo. Manejo integral de la fertilidad del suelo. Edit. Guadalupe Ltda. Bogotá. p.111-119.

MEDINA, A. 2003. Síntomas de deficiencias minerales en las plantas. En: Soc. Col. de la Ciencia del Suelo. Manejo integral de la fertilidad del suelo. Edit. Guadalupe Ltda.. Bogotá. p.23-30.

MEJÍA, J.; OSORIO, F. 1983. Inhibición de la absorción de selenio en trigo, maíz, fríjol, avena y Angleton. Tesis I. A. U. N. Colombia. Facultad de Agronomía. Bogotá, D.E. 148p.

MEJÍA, J. 1998. Degradación de suelos y cultivos por contaminación con elementos traza y pesados derivados de diversas fuentes en Colombia. En: Soc. Col. de la Ciencia del Suelo. Encuentro Nacional de Labranza de Conservación. Edit. Guadalupe Ltda. Bogotá. p.97-144.

MÉNDEZ, L.; RODRÍGUEZ, L.; PALACIOS, S. 2000. Impacto del agua de riego con aguas contaminadas, evaluado por la presencia de metales pesados en suelos. Terra Latinoam. (México). 18(4):277288.

MENGEL, K.; KIRKBY, A. 1979. Principles of plant nutrition. Edit. Internacional Potash Institue, Berna (Suiza). 593p.

MOLINA, G.; FRYE, C. 1982. Selección de métodos químicos para la evaluación de elementos menores en suelos algodoneros de Colombia. Suelos Ecuatoriales. 12(1):200-211. 
MOLAS, F.; BARAN, S. 2004. Relationship between the chemical form of nickel applied to the soil and the uptake and toxicity to barley plants. Geoderma. 122:247-255

MOTTA, B. 1976. Efecto de dos métodos de fertilización con molibdeno en el rendimiento de la coliflor en un suelo de la serie Mosquera. Tesis M.Sc. Programa de Estudios para Graduados ICA-UN. Bogotá. 88p.

MUCHAWETI, M.; BIRKETT, J.; CHINYANGA, E.; LESTER, J. 2006. Heavy metal content of vegetables irrigated with mixtures of waste water and sewage sludge in Zimbabwe. Agric. Ecos. Environ. 112:41-48.

MUNÉVAR, F. 1999. Revisión de conceptos básicos sobre el suelo. En: Memorias: Ciclo de cursos de actualización de conocimientos con aplicación en palma africana de aceite. CENIPALMA. Santa Fe de Bogotá, D.C. p.4-23.

ORTIZ, G. 1998. Fertilización de la soya con micronutrimentos en suelos del Valle del Cauca. Ed. Selon, F. Actualidad y futuro de los micronutrientes en la agricultura. $2^{a}$ ed. Prolabo Ltda. Palmira. p.157171.

PETERSON, R.G.; CALVIN, L.D. 1965. Sampling. In: Black, C.A. et al. (Ed.) Methods of soil analysis. Am. Soc. Agronomy (EEUU). p.54-72.

PUENTES, G.; RODRÍGUEZ, C. 1983. Determinación de molibdeno en un inceptisol de Sutamarchán e inhibición de su absorción en Avena sativa L. Tesis Químico. Facultad de Ciencias. U. N. Colombia. Bogotá, 149p.

SALISBURY, F.; ROSS, C. 1994. Fisiología Vegetal. Grupo Edit. Interamericana S.A., México. 759p.
TAMAYO, A.; HINCAPIÉ, M. 2002. Micronutrimentos en cítricos y relación con su contenido en suelos de Caicedonia, Valle del Cauca.En: Resúmenes X Congreso Nacional de la Ciencia del Suelo. Medellín. Soc. Col. de la Ciencia del Suelo. p.21-24.

TISDALE, S.; NELSON, W.; BEATON, J. 1993. Soil fertility and Fertilizers. Edit. McMillan Co. N.Y. p.350-413.

TORRES, C.; JIMÉNEZ, M.; LORA, R. 1978. Determinación de selenio en suelos y material vegetal e inhibición de su absorción en Ryegrass (Lolium multiflorum). Suelos Ecuatoriales 9(2):218-223.

TURNER, J. 1978. Boron in agricultura. U.S. Borax a member of RTZ group. N.Y. 41p.

VARGAS, C.; BALLESTEROS, I.; TRIANA, P. 2002. Evaluación de extractantes para la determinación de boro disponible en suelos. Suelos Ecuatoriales. 32:84-89.

VILLAR, H.; LORA, R. 1992. Disponibilidad de elementos menores en algunos suelos tabacaleros de Santander. Suelos Ecuatoriales. 12(1):310-327.

WONG, J.; LI, K.; ZHON, L.; SELVAM, A. 2006. The sorpsion of $\mathrm{Cd}$ and $\mathrm{Zn}$ by different soils in presence of dissolved organic matter from sludge. Geoderma. (in press).

ZAPATA, R. 2005. Función de la materia orgánica sobre la movilidad iónica. Suelos Ecuatoriales. 35(1):2335.

ZHELYAZKOV, V.; CRAKER, L.; XING, B. 2006. Effects of $\mathrm{Cd}, \mathrm{Pb}$ and $\mathrm{Cu}$ on growth and essential of oil contents in dill, peppermint, and basil. Environm. Exp. Bot. 58:9-16.

Recibido: Julio 26 de 2006

Aceptado: Marzo 1 de 2007 